\title{
A Method of WLAN Indoor Location Based on MMDM-Boost
}

\author{
Xiaoyi $\mathrm{Li}^{1, \mathrm{a}}$, Ke Wen ${ }^{2, \mathrm{~b}}$
}

\begin{abstract}
${ }^{1,2}$ Key Laboratory of Fiber Optic Sensing Technology and Information and Processing(Wuhan University of Technology), Ministry of Education, School of Information Engineering, Wuhan, Hubei Province,China
\end{abstract}

a1115304200@qq.com

\begin{abstract}
Keywords: WLAN; indoor positioning; AP signal strength; fingerprint; Multiple Mixed Distribution Model (MMDM); Adaoost
\end{abstract}

\begin{abstract}
Towards the problem of the time-varying AP signal strength degrades the indoor positioning accuracy in Wireless Local Area Network (WLAN), a WLAN indoor positioning method based on multiple mixed distribution model (MMDM) and Adaboost algorithm is adopted. Firstly, in order to describe the probability density distribution of AP signal strength accurately and improve the system suitability, the proposed method employs Gaussian mixture model, Binomial mixture model and Poisson mixture model to make up the MMDM and construct the fingerprint database. Secondly, in order to avoid the insufficient training of positioning model caused by lacking of training samples, the Adaboost is used to comprise the weak classifier based multiple MMDM into a strong classifier and improve the generalization ability of the system. Lastly, the mapping relation between fingerprint data and real position is also built by Adaboost classifier in online positioning stage. The experimental results show that the proposed method is superior to several indoor positioning algorithms with better time shifting adaptability and positioning accuracy.
\end{abstract}

\section{Introduction}

Due to the indoor location technology based on GPS and cellular network cannot satisfy the needs of indoor location service such as personalized information transmission, medical service, home security and target discovery. Indoor location system based on wireless local area network (WLAN) has been widespread concern because of its high positioning accuracy and stability.

In the field of indoor positioning, the GMM is generally used to reflect the spatial and temporal characteristics of AP signal intensity to construct the location fingerprint. Chen Miao et al ${ }^{[1]}$ pointed out that the selection of the probability density function of signal intensity distribution has a critical impact on system performance due to the serious multipath interference in the indoor environment. Cui Wei et al ${ }^{[2]}$ used particle swarm optimization algorithm to optimize the EM algorithm, they proposed a GMM based non-line-of-sight location algorithm to overcome the non-line-of-sight, effectively improving the positioning accuracy with excellent stubbornness. Qiao Shaojie et al. ${ }^{[3]}$ used the GMM to construct the complex motion model, and calculate the probability distribution of different motion patterns, and then the Gaussian process regression method was used to predict the most possible moving trajectories. Ndeye et al. ${ }^{[4]}$ proposed a binary GMM to detect and exclude anomaly measurements in the location fingerprint database. Alfakih et al ${ }^{[5]}$ used the GMM to approximate the probability distribution of received WIFI signal strength, thus locating the mobile position. However, the propagation path of the AP transmission signal is often blocked by objects, such as buildings and people moving in the practical application environment, resulting in the wave signal travels by refraction, reflection and other non-line-of-sight propagation. Hu Qiao-ling et al. ${ }^{[6]}$ proposed an indoor positioning method by adopting Gaussian distribution or logarithmic distribution in most areas to describe signal fingerprint characteristics, by adopting discrete distribution in special area to match the signal samples, improving the adaptability of system. Wang Saiwei et al ${ }^{[7]}$ used improved discrete probability method to construct the position fingerprint, he use the curve fitting to reflect the actual location information, thereby improving the positioning accuracy and adaptability. Milutin et al. ${ }^{[8]}$ use the discrete random variable to represent the path loss parameter and use the EM algorithm to estimate the path loss effect of the WIFI signal, with the average 
positioning error below $4.5 \mathrm{~m}$ is achieved. Therefore, it is an effective method to improve the applicability of the system localization by introducing the Gaussian mixture model and binomial distribution model to represent the spatial distribution of position fingerprints in most spatial and spatial locations respectively, by introducing the Poisson distribution with the random variable which is proportional to the time or space length to reflect the time variation of position fingerprint in a few spatial locations.

However, concerning the effects of multipath, line-of-sight and personnel movement in a practical environment, the spatio-temporal characteristics of AP signal strength ${ }^{[9-10]}$ cannot be reflected by the fingerprint data with small sample size efficiently. Adaboost is used to form a strong classifier by changing the weight of the training samples to update the weight of several weak classifiers, the weak classifier based on appropriate sample size only need to meet some corresponding requirements ${ }^{[11]}$, the result of a strong classifier with high accuracy we can obtain. For example, David et al. ${ }^{[12]}$ used the decision tree as the weak classifier to construct the indoor positioning system with advantages of excellent localization accuracy and lower algorithm complexity. Feng $\mathrm{Yu}$ et al. ${ }^{[13]}$ proposed an improved Adaboost algorithm to obtain good positioning accuracy by removing the error points in the location fingerprint database, thus improving the indoor positioning accuracy. Oscar et al. ${ }^{\text {[14] }}$ proposed a WiFi-Boost classification algorithm based on the feature combination between AP pairs, which minimized the adverse effects of WLAN network structure change and AP failure that could not be predicted. However, the traditional Adaboost algorithm architecture is used to solve the binary classification problem. In view of this, Yang Xinwu et al. ${ }^{[15]}$ proposed an Adaboost method based on SAMME.R, which not only guarantees the availability of weak classifiers, but also guarantees the quality of weak classifiers by resampling. Therefore, in this paper, we use the Adaboost method based on SAMME.R to mix the weak classifiers based on MMDM into strong classifiers, the experimental results show that the MMDM-Boost method proposed is superior to several indoor positioning algorithms with better time shifting adaptability and positioning accuracy.

\section{Elementary Theory}

Multiple Mixed Distribution Model. In order to solve the problem that the complex data cannot be effectively fitted by a single distribution model, the researchers proposed a hybrid distribution model based on different distributions to fit the complex data of different application domains ${ }^{[16]}$. In general, the mixed distribution model is constructed by joint distribution based on different distributions. In this paper, we propose the MMDM method based on Gaussian distribution model and discrete distribution model to describe the probability distribution of AP signal strength in indoor positioning system. The Gaussian mixture model is based on the Gaussian distribution, the discrete distribution model includes a mixture of binomial distribution based on binomial distribution model and a mixture of Poisson distribution based on Poisson distribution model. The binomial distribution is a discrete probability distribution of the number of successes in $n$ times independent experiments. It is said that each binomial distribution model can be regarded as independent experiments in the mixed binomial distribution model. According to the frequency histogram of the fingerprint samples, the occurrence frequency $\mathrm{k}$ and the number $\mathrm{m}$ of different frequencies of each location fingerprint sample are obtained. A mixed binomial distribution model contained $\mathrm{m}$ binomial distributions is designed to express the probability distributions of different fingerprint samples respectively when the frequency is $\mathrm{K}$. At the same time, the EM algorithm is used to estimate the probability value p of each binomial distribution model. The result will reflect the probability that the location fingerprint sample appeared frequency $\mathrm{k}$. This will satisfy the condition that the probabilities of result and its opposite result are fixed. Poisson distribution is a limiting case of the binomial distribution, it is used to simulate low probability events because of the regulation at uncommon time can be reflected by Poisson distribution. The condition of applying the mixed Poisson distribution is the same as the condition of mixed binomial distribution in the indoor location application. It is worth noting that the key feature of the Poisson distribution that the value of a random variable is proportional to the length of time or space. Considering the indoor positioning system based on RSSI has the characteristics of positioning 
accuracy drifted with time, the mixed binomial distribution is introduced to simulate the change of signal strength in the long time of the collected fingerprint data.

An Introduction to Adaboost Algorithm Based on SAMME.R. The SAMME.R method proposed by Yang Xinwu et al., is a multi-class classification method, For there are two problems of the Adaboost.M1 algorithm, (1) the classification of weak classifier based on the Adaboost.M1 is difficult to achieve; (2) It is hard to guarantee the weight of the correct classified sample based on the SAMME algorithm larger than the weight of the incorrect classified sample, so the final classification cannot be guaranteed. In the multi-class problems, the correct rate of random guess is only $1 / \mathrm{K}(\mathrm{k}$ means the number of sample classes), and the correct rate of each weak classifier greater than $1 / 2$ cannot be guaranteed by adopting the Adaboost.M1 algorithm, thus obtaining the strong classifier is a difficult work because of the number of weak classifiers is not enough. Zhu et al. ${ }^{[11]}$ proposed a SAMME method for multi-classification problem. The principle of this method is designed to reduce the requirement of the weak classifier from $1 / 2$ to $1 / \mathrm{k}$ by changing the weighting formula of the sample weight, which is a good guarantee to form the final strong classifier. However, in some practical multi-classification problems, the actual effect of SAMME method is not as good as the method of Adaboost.M1. Although the SAMME method relaxes the requirement of the accuracy of weak classifier, it does not pay attention to the quality of weak classifier, so the classification result of the final strong classifier cannot be guaranteed. SAMME.R is an improved Adaboost method. The core of the SAMME.R method is designed to judge the classification result of the weak classifier in each iteration process. The classification that the sum of the sample weights is greater than the sum of the weights of misclassification samples in the same class can be ensured. When the condition is met, the next iteration is performed, and the weak classifier is re-trained. In this step, when the number of iterations tends to infinity, the number of labels correctly classified in each category is more than the number of misclassified labels, and the error rate of the strong classifier tends to zero.

\section{Indoor Location Algorithm of WLAN Based on MMDM-Adaboost}

Location Fingerprint Construction Based on MMDM. The MMDM is constructed by using the $L_{1}$ order Gaussian mixture model, the $L_{2}-L_{1}$ order mixed binomial distribution model and the $L-L_{2}$ order mixed Poisson distribution model, and the fingerprint data $X=\{x 1, x 2, \ldots, x n\}$ in the d-dimensional space of the probability distribution can be fitted, as the expression shown in (1).

$$
\mathrm{G}\left(x_{i}, \theta\right)=\sum_{j=1}^{L_{1}} \alpha_{j} g\left(x_{i} ; u_{j}, \sigma_{j}\right)+\sum_{j=L_{1}}^{L_{2}} \alpha_{j} b\left(x_{i} ; p_{j}\right)+\sum_{j=L_{2}}^{L} \alpha_{j} t\left(x_{i} ; \gamma_{j}\right)
$$

$\theta$ is the parameter of Gaussian mixture distribution probability density function $\left[\alpha_{j}, u_{j}, \sigma_{j}, p_{j}, \gamma_{j}\right]$, $u_{j}$ and $\sigma_{j}$ are the expectation and variance of the jth single Gaussian model, $p_{j}$ is the probability of occurrence of the fingerprint data sample $x_{i}$ in the jth binomial distribution model, $\gamma_{j}$ is the probability of occurrence of the location fingerprint data sample $x_{i}$ in the jth Poisson distribution model, $\alpha_{j}$ denotes the weight of the Gaussian model, binomial distribution model or Poisson distribution model in the multi-mixed distribution model, and the sum of all $\alpha_{j}$ is 1 . In order to obtain the probability density function parameter $\theta$ which maximizes the probability of the location fingerprint data $\mathrm{X}$ to be the joint distribution model $\mathrm{G}\left(x_{i}, \theta\right)$.As shown in equation (2).

$$
\mathrm{J}(\theta)=\sum_{i=1}^{n} \ln \left[\sum_{j_{1}=1}^{L_{1}} \alpha_{j_{1}} g\left(x_{i} ; u_{j_{1}}, \sigma_{j_{1}}\right)+\sum_{j_{2}=L_{1}}^{L_{2}} \alpha_{j_{2}} b\left(x_{i} ; p_{j_{2}}\right)+\sum_{j_{3}=L_{2}}^{L} \alpha_{j_{3}} t\left(x_{i} ; \gamma_{j_{3}}\right)\right]
$$

The EM algorithm is used to estimate the parameters of probability density function, and the implicit variable $Z_{j}$ is introduced to represent the probability of each fingerprint data sample generated by the MMDM. The steps of EM algorithm are described below:

a. In step E, probability $\beta_{j}\left(x_{i}\right)$ that the $j$ th single MMDM generated is obtained by adopting the Bayes theorem to compute the sample $x_{i}$ of fingerprint data. The process is given by the formula (3). 


$$
\beta_{j}\left(x_{i}\right)=\left\{\begin{array}{c}
p\left(Z_{j} \mid x_{i}\right)=\frac{p\left(Z_{j}\right) p\left(x_{i} \mid z_{j}\right)}{p\left(x_{i}\right)}=\frac{\alpha_{j} g\left(x_{i} ; u_{j}, \sigma_{j}\right)}{\mathrm{G}\left(x_{i}, \theta\right)}, 0<j \leq L_{1} \\
p\left(Z_{j} \mid x_{i}\right)=\frac{p\left(z_{j}\right) p\left(x_{i} \mid z_{j}\right)}{p\left(x_{i}\right)}=\frac{\alpha_{j} \mathrm{~b}\left(x_{i}, p_{j}\right)}{\mathrm{G}\left(x_{i}, \theta\right)}, L_{1}<j \leq L_{2} \\
p\left(Z_{j} \mid x_{i}\right)=\frac{p\left(z_{j}\right) p\left(x_{i} \mid z_{j}\right)}{p\left(x_{i}\right)}=\frac{\alpha_{j} \mathrm{t}\left(x_{i}, \gamma_{j}\right)}{\mathrm{G}\left(x_{i}, \theta\right)}, L_{2}<j \leq L
\end{array}\right.
$$

b. In step M, the partial derivatives of $u_{j}, \sigma_{j}, p_{j}, \gamma_{j}$ is shown in the formulas (4) - (7).

$$
\begin{aligned}
& J_{u_{j}}(\theta)=\sum_{i=1}^{n} \frac{\alpha_{j_{1}} g\left(x_{i} ; u_{j}, \sigma_{j}\right)}{\mathrm{G}\left(x_{i}, \theta\right)} \frac{x_{i}-u_{j}}{\sigma_{j}^{2}}=\sum_{i=1}^{n} \beta_{j}\left(x_{i}\right)\left(\frac{x_{i}-u_{j}}{\sigma_{j}^{2}}\right), 0<j \leq L_{1} \\
& \mathrm{~J}_{\sigma_{\mathrm{j}}}(\theta)=\sum_{\mathrm{i}=1}^{\mathrm{n}} \beta_{\mathrm{j}}\left(\mathrm{x}_{\mathrm{i}}\right)\left[\frac{\left(\mathrm{x}_{\mathrm{i}}-\mathrm{u}_{\mathrm{j}}\right)^{\mathrm{T}}\left(\mathrm{x}_{\mathrm{i}}-\mathrm{u}_{\mathrm{j}}\right)}{\sigma_{\mathrm{j}}^{3}}-\frac{\mathrm{d}}{\sigma_{\mathrm{j}}}\right], 0<\mathrm{j} \leq \mathrm{L}_{1} \\
& J_{p_{j}}(\theta)=\sum_{i=1}^{n} \frac{\alpha_{j} \mathrm{~b}\left(x_{i}, p_{j}\right)}{\mathrm{G}\left(x_{i}, \theta\right)}\left(\frac{k_{j}}{p_{j}}-\frac{n-k_{j}}{1-p_{j}}\right)=\sum_{i=1}^{n} \beta_{j}\left(x_{i}\right)\left(\frac{k_{j}}{p_{j}}-\frac{n-k_{j}}{1-p_{j}}\right), L_{1}<j \leq L_{2} \\
& J_{\gamma_{j}}(\theta)=\sum_{i=1}^{n} \frac{\alpha_{j} \mathrm{t}\left(x_{i}, \gamma_{j}\right)}{\mathrm{G}\left(x_{i}, \theta\right)}\left(\frac{k_{j}}{\gamma_{j}}-1\right)=\sum_{i=1}^{n} \beta_{j}\left(x_{i}\right)\left(\frac{k_{j}}{\gamma_{j}}-1\right), L_{2}<j \leq L
\end{aligned}
$$

$\mathrm{N}$ indicates the number of position fingerprint data samples that a binomial distribution model contains. Let the above four equations be 0 , the results are shown in (8) - (11).

$$
\begin{aligned}
u_{j} & =\frac{\sum_{i=1}^{n} \beta_{j}\left(x_{i}\right) x_{i}}{\sum_{i=1}^{n} \beta_{j}\left(x_{i}\right)}, 0<j \leq L_{1} \\
\sigma_{j} & =\frac{1}{d} \frac{\sum_{i=1}^{n} \beta_{j}\left(x_{i}\right)\left(x_{i}-u_{j}\right)^{T}\left(x_{i}-u_{j}\right)}{\sum_{i=1}^{n} \beta_{j}\left(x_{i}\right)}, 0<j \leq L_{1} \\
p_{j} & =\frac{\sum_{i=1}^{n} \beta_{j}\left(x_{i}\right) k_{j}}{\sum_{i=1}^{n} \beta_{j}\left(x_{i}\right) n}, L_{1}<j \leq L_{2} \\
\gamma_{j} & =\frac{\sum_{i=1}^{n} \beta_{j}\left(x_{i}\right) k_{j}}{\sum_{i=1}^{n} \beta_{j}\left(x_{i}\right)}, L_{2}<j \leq L
\end{aligned}
$$

For solving the weight $\alpha_{j}$ in the MMDM, $\alpha_{j}$ is solved by the Lagrangian multiplier method. a new likelihood function $J_{\text {new }}(\theta)$ for the joint distribution model is defined, as is shown in formula (12).

$$
J_{\text {new }}(\theta)=J(\theta)+\lambda\left(1-\sum_{j=1}^{L} \alpha_{j}\right)
$$

The new likelihood function $J_{\text {new }}(\theta)$ is used to derive the derivative of $\alpha_{j}$.As is shown in (13).

$$
J_{\alpha_{j}}(\theta)=\left\{\begin{array}{c}
\sum_{i=1}^{n} \frac{g\left(x_{i} ; u_{j}, \sigma_{j}\right)}{\mathrm{G}\left(x_{i}, \theta\right)}-\lambda=\frac{1}{\alpha_{j}} \sum_{i=1}^{n} \beta_{j}\left(x_{i}\right)-\lambda, 0<j \leq L_{1} \\
\sum_{i=1}^{n} \frac{b\left(x_{i} ; p_{j}\right)}{\mathrm{G}\left(x_{i}, \theta\right)}-\lambda=\frac{1}{\alpha_{j}} \sum_{i=1}^{n} \beta_{j}\left(x_{i}\right)-\lambda, L_{1}<j \leq L_{2} \\
\sum_{i=1}^{n} \frac{t\left(x_{i} ; \gamma_{j}\right)}{\mathrm{G}\left(x_{i}, \theta\right)}-\lambda=\frac{1}{\alpha_{j}} \sum_{i=1}^{n} \beta_{j}\left(x_{i}\right)-\lambda, L_{2}<j \leq L
\end{array}\right.
$$

The partial derivative of $\lambda$ is determined by the new likelihood function $J_{\text {new }}(\theta)$ in equation (14). $J_{\lambda}(\theta)=\sum_{j=1}^{L} \alpha_{j}-1$

Let the equations (13) and (14) be 0 , and the formula for $\alpha_{j}$ is as shown in equation (15)

$$
\alpha_{j}=\frac{\sum_{i=1}^{n} \beta_{j}\left(x_{i}\right)}{n}, 0<j \leq L
$$

Location Fingerprint Construction Based on MMDM. The MMDM-Boost-based WLAN indoor location method is divided into offline phase and on-line phase. Two fields are mainly completed by the construction of the location fingerprint database based on the MMDM and the training of the strong classification classifier based on SAMME.R in the offline phase. The offline phase is accomplished by the following steps:

Step 1: Initial sample weights $w_{i}^{1}=1 / n, i=1,2, \ldots, n$, for the global sample set $\mathrm{Y}=[\mathrm{y} 1, \mathrm{y} 2, \ldots, \mathrm{yn}], \mathrm{n}$ is the total number of samples, $y_{i}$ represents actual sample for each category.

Step 2: for $t=1,2, \ldots T$, $T$ is the number of iterations of Adaboost method, perform steps (1) - (6):

(1) The whole sample is divided into training samples and testing samples by resampling strategy.

(2) Based on the MMDM, the location fingerprint is constructed by selecting training samples. The construction process is implemented as follows: (2a) The weight $\alpha_{j}=\frac{1}{\mathrm{~L}}$ of each model in the MMDM, $\mathrm{L}$ value stands for the training sample category number. Therefore, the proportions of the single Gaussian model, the binomial distribution model and the Poisson distribution model are set as 3: 1: 1 
in the MMDM. The clustering centers of each location fingerprint category are determined by applying the Kmeans algorithm to calculate the values of $u_{j}, \sigma_{j}, p_{j}$ and $\gamma_{j}$. (2b) The step 1 is calculated by the parameter value $\theta, \beta_{\mathrm{j}}\left(\mathrm{x}_{\mathrm{i}}\right)$ is calculated according to the formula (3), where $0<j \leq L, \quad 1 \leq \mathrm{i} \leq \mathrm{n}$. (2c) The next iteration is performed by using $\beta_{\mathrm{j}}\left(\mathrm{x}_{\mathrm{i}}\right)$ calculated in step 2 , and $\theta$ is updated according to (8) - (11) and (15). (2d) The new value of $\theta$ is obtained in step 3, the value of $J(\theta)$ is calculated according to equation (2), which is compared to the value of $J(\theta)$ obtained from the previous iteration, and stops the algorithm if the difference is less than the threshold Iteration, the mapping of the training samples to the spatial position is performed by step 5 . Otherwise, step 2 is performed for the next iteration. (2e) The probabilities of generating each training sample for each single distribution model in the MMDM are taken as the single distribution model with the highest probability, and then the whole training sample is divided into different categories.

(3) The MMDM constructed in step (2) is used as the weak classifier to classify the test samples one by one. The test sample contains k categories, each category included in the test sample is polled, and the following two steps are performed to calculate the sum of the weighting of each category to the right sample and the wrong sample. (3a) for $\mathrm{k}=1,2, \ldots, \mathrm{K}$, for $\mathrm{j}=1,2, \ldots, \mathrm{K}$

$\mathrm{r}_{\mathrm{tkj}}=\sum_{\mathrm{i}=1}^{\mathrm{m}} \mathrm{w}_{\mathrm{i}}^{\mathrm{t}}\left[\mathrm{y}_{\mathrm{i}}=\mathrm{k}, \mathrm{h}_{\mathrm{t}}\left(\mathrm{x}_{\mathrm{i}}\right)=\mathrm{j}\right]$

(3b) It's necessary to determine whether the sum of the correctly classified sample weights in each class is greater than the sum of the sample weights of the misclassified samples, $r_{\text {tkj }}\left[h_{t}\left(x_{i}\right)=j\right] \geq$ $r_{t k j}\left[h_{t}\left(x_{i}\right) \neq j\right]$. If the condition is met, then the next iteration is performed. Conversely, step 2 is performed until the condition is satisfied.

(4) The current iteration times under the multiple mixed distribution model error rate is computed: $\varepsilon_{\mathrm{t}}=\sum_{\mathrm{i}=1}^{\mathrm{n}} \mathrm{w}_{\mathrm{i}}^{\mathrm{t}}\left[\mathrm{h}_{\mathrm{t}}\left(\mathrm{x}_{\mathrm{i}}\right) \neq \mathrm{y}_{\mathrm{i}}\right]$

(5) The weight of the MMDM under the current iteration times is computed:

$\alpha_{\mathrm{t}}=\ln \frac{\varepsilon_{\mathrm{t}}}{1-\varepsilon_{\mathrm{t}}}+\ln (\mathrm{k}-1)$

(6) The test sample weights $\mathrm{w}_{\mathrm{i}}^{\mathrm{t}+1}$ are updated and all sample weights are normalized:

$\mathrm{w}_{\mathrm{i}}^{\mathrm{t}+1}=\mathrm{w}_{\mathrm{i}}^{\mathrm{t}} * \exp \left(\alpha_{\mathrm{t}} *\left[\mathrm{~h}_{\mathrm{t}}\left(\mathrm{x}_{\mathrm{i}}\right) \neq \mathrm{y}_{\mathrm{i}}\right]\right)$

Step 3: All iterations are completed, the final strong classifier is

$\mathrm{h}_{\mathrm{f}}(\mathrm{x})=\operatorname{argmax} \sum_{\mathrm{t}=1}^{\mathrm{T}} \alpha_{\mathrm{t}} *\left[\mathrm{~h}_{\mathrm{t}}\left(\mathrm{x}_{\mathrm{i}}\right) \neq \mathrm{y}_{\mathrm{i}}\right]$

In online phase. The mapping relationship between the fingerprint data and the actual spatial position are established by the strong classifier. Firstly, the fingerprint tested samples are brought into T multiple MMDM, and the corresponding category of the maximum probability value is chosen as the position of the fingerprint tested samples according to the probability vector of each mixed model obtained. Finally, input the weak classifier classification result to the strong classifier, and the classification result with the largest sum of the weights is taken as the final estimation of the tested point. The flow chart of indoor positioning algorithm based on MMDM-Boost shown in Figure 1.

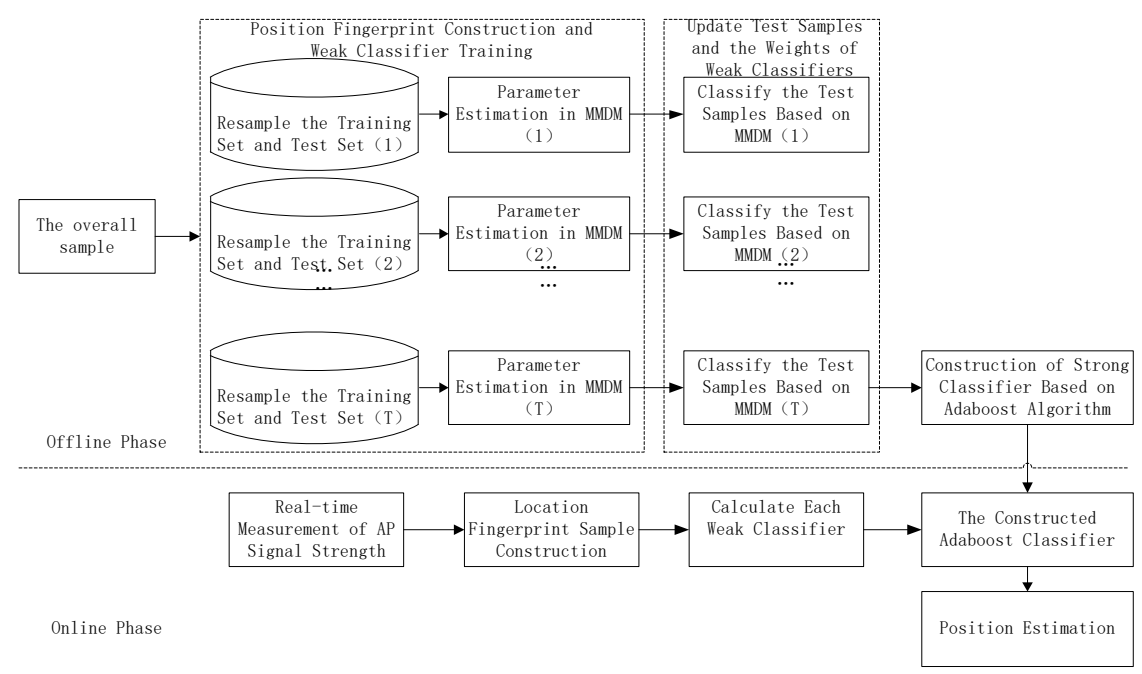

Figure 1. MMDM-Boost Algorithm Flow Chart 


\section{Experimental Results and Analysis}

Introduction to Experimental Data. Experimental environment shown in Figure 2, due to the limited use of school area, A, B and C three regions are selected to collect the reference point and RSS signals respectively. Among them, A area is the student laboratory, put tables and chairs, bookcases and other office supplies, B area is the linear floor corridor, C area is the elevator waiting area. Triangle shown in the figure 2 indicates the selection of 37 RSS signal collection points, each signal acquisition point interval of 2 meters. The RSS signal sampling software is used to collect the RSS signal samples for 20 days, and 50 samples of RSS signal are collected at each signal collection point. The sampling frequency is 1 time / second. After data sampling, 1000 samples of RSS signal were collected at each signal collection point, and the total sample size was 37000 . The whole sample was divided into 10 parts by using 10 -fold cross validation, 9 of which were used as location fingerprints at different reference points to train the location model in the offline phase, the remaining one was used as the location fingerprint at different reference points in the online phase to estimate the location model performance, an average of 100 results was used as the positioning performance of the algorithm. In order to compare the positioning performance of different algorithms in different positioning accuracy, the research group finished the arrangement of 37 RSS signal acquisition points, divided the signal acquisition points into different location areas, such as 2 meters, 6 meters, 8 meters, 10 meters and 12 meters and other six kinds of positioning accuracy. In this paper, seven kinds of indoor localization methods, such as KNN, libSVM, GMM, KNN-Boost, libSVM-Boost, GMM-Boost and MMDM-Boost are implemented respectively. The experimental results obtained by different localization algorithms are described in Section 4.2.

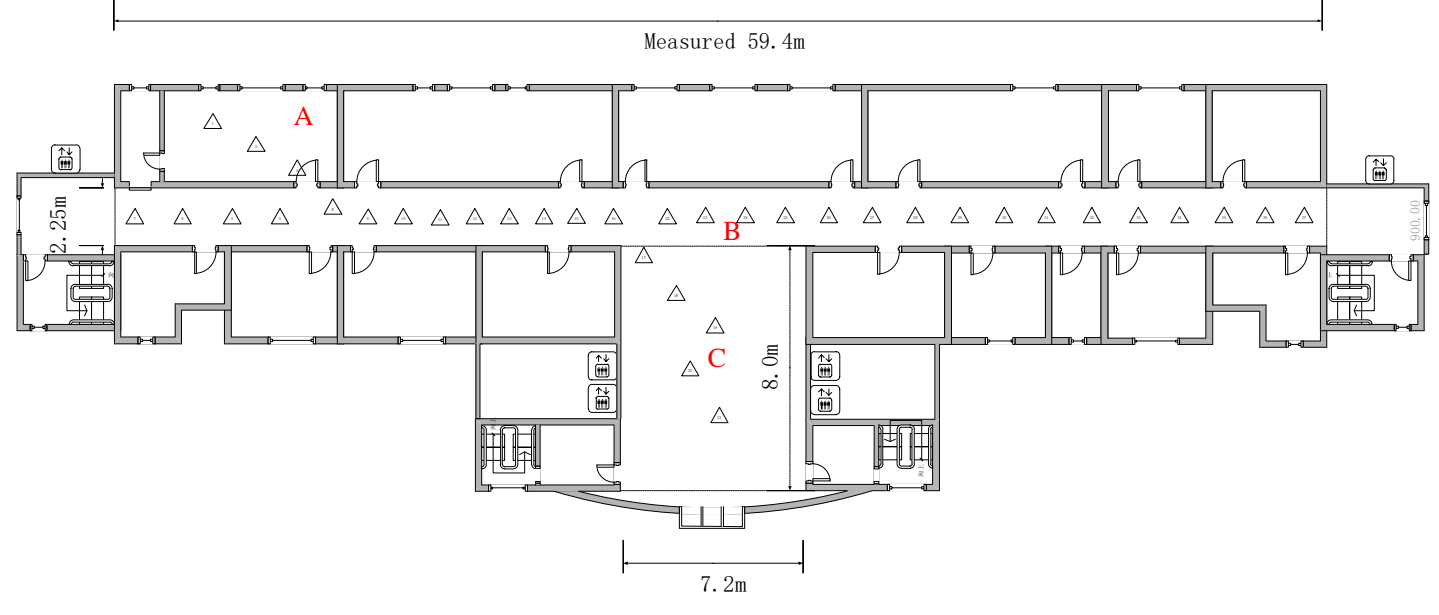

Figure 2. Experimental Environment Diagram

Experimental Results and Analysis. In the experiment, the positional fingerprint classification effect of different classification algorithms is evaluated by the following location accuracy rate $t_{p}$, which is defined as (21).

$$
t_{p}=\frac{N_{T P}}{N_{T P}+N_{F N}}
$$

Among them, $N_{T P}, N_{F N}$ are the number of samples correctly identified by the algorithm and the number of samples incorrectly identified by the algorithm. In this paper, two groups of experiments were organized to evaluate the effectiveness of the algorithm:

(1) In order to compare the positioning effect of the MMDM-Boost and other algorithms, the other six kinds of localization such as KNN, libSVM, GMM, KNN-Boost, libSVM-Boost and GMM-Boost are implemented. First, in the offline phase, the localization model is constructed by the training data set. Then, the parameters of excellent positioning performance are set as actual parameter values, and the positioning operation of the test set is applied in the online phase. Finally, the accuracy of classification of the test data set is calculated for different localization accuracy of each algorithm, and the cumulative error probability distribution is given. Figure 3 shows the cumulative probability distributions of different localization errors for different algorithms. The following conclusions can be drawn: (1a) Compared with KNN, libSVM and GMM algorithm, the 
localization accuracy of different localization algorithms can be improved by the combination of Adaboost method. (1b) The location accuracy of MMDM-Boost positioning method is higher than the other localization algorithms under different positioning accuracy. It is shown that the probability distribution of AP signal intensities in most regions and special regions can be more abundantly reflected by the combination of MMDM. Compared with the Gaussian distribution model, the location fingerprints constructed based on the MMDM are more consistent with the time-varying nature of the AP signal intensity in the indoor space.

(2) The relationship between the location accuracy of different localization methods and the number of weak classifiers is compared. As shown in Fig. 4, for KNN-Boost and libSVM-Boost localization methods, the localization accuracy increases with the increase of the number of weak classifiers, indicating that with the increase of the number of classifiers, the generalization ability of positioning method is improved. with this feature is consistent with the Adaboost method, that is, when the error rate of a single weak classifier is less than $1 / 2$, and the error is uncorrelated among multiple weak classifiers, the error rate of the strong classifier will decreasing monotonically with the number of weak categories increase. In addition, for MMDM-Boost and GMM-Boost localization algorithms, when the number of classifiers increases to about 10 , the time complexity of the two localization algorithms increases, that is, as the number of weak classifiers based on MMDM or GMM increases, The weight of the training samples is no longer updated, so that the classification error rate can not be reduced by the strong classifier continually, indicating that the Adaboost positioning method based on MMDM and GMM has better convergence. By the measure of MMDM model and GMM model constructed position fingerprints, the spatial and temporal characteristics of the RSS signal in the indoor space can be better reflected.

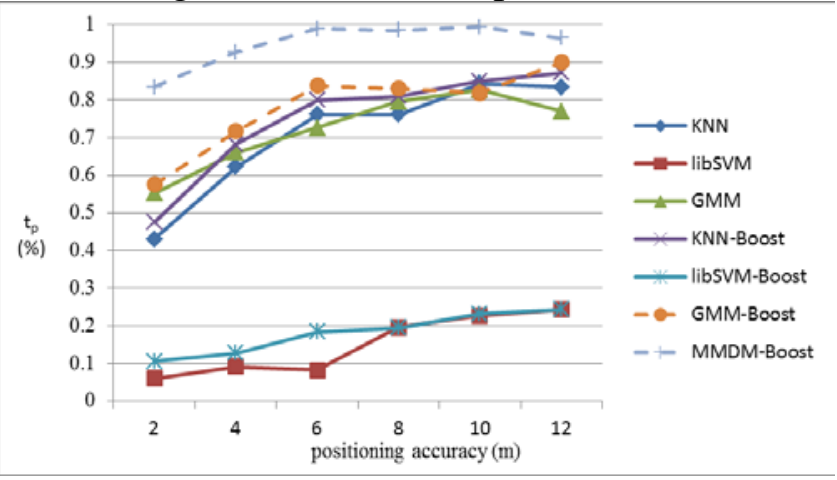

Figure 3. Cumulative probability distribution

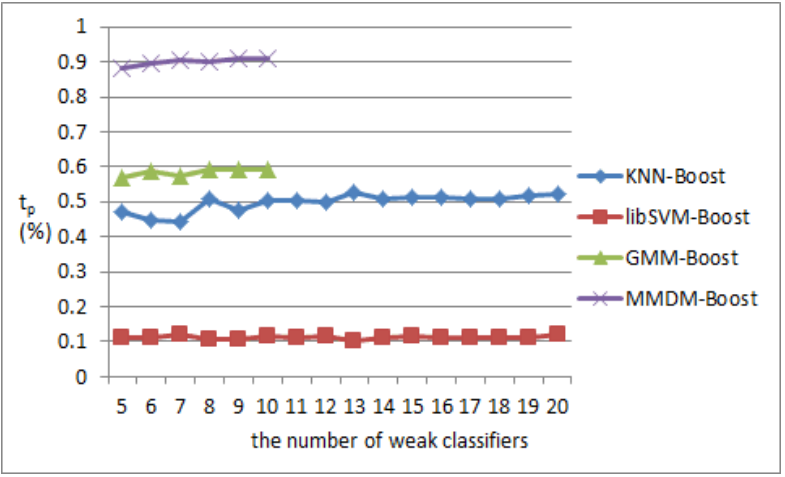

Figure 4. The positioning accuracy under 2 meters

\section{Conclusions}

In this paper, we propose a WLAN indoor location method based on multiple mixed distribution model (MMDM) and Adaboost algorithm based on SAMME.R for indoor positioning system. The objective of this method is to construct a multi-mixed model by Gaussian mixture model, binomial mixture model and Poisson mixture model, and to construct the position fingerprints by using the MMDM as the weak classifier, and to complete the strong classification of Adaboost method based on SAMME.R Training. In the online positioning phase, the constructed strong classifier method establishes the mapping relationship between the position fingerprint data and the actual spatial position. The experimental results show that compared with other localization methods, MMDM-Boost indoor positioning method can obtain higher accuracy of positioning. At the same time, with the increase of the number of weak classifiers, the MMDM-Boost localization method has better generalization ability and algorithm convergence under the 2 meter positioning accuracy.

In this paper, the algorithm proposed also has some shortcomings. On one hand, in the process of obtaining the strong classifier, the Adaboost method based on SAMME.R is not easy to acquire the classifier condition after repeated training, which increases the time complexity of the algorithm.On the other hand, the experiment scene is ideal, and the spatio-temporal variation of RSS signal is less affected by multipath, line-of-sight effect and personnel movement, which results in less location area with discrete distribution. Therefore, in the future research, we must not only solve the time 
complexity of the algorithm, but also need to further try more complex experimental environment, and then prove the rationality and applicability of this method.

\section{References}

[1] Miao C. WLAN indoor location system based on multi-Gaussian mixture model[J]. Journal of Huazhong University of Science \& Technology, 2012.

[2] Wei C. GMM-based localization algorithm under NLOS conditions[J]. Journal on Communications, 2014.

[3]QIAO Shao-Jie,JIN Kun,HAN Nan, et al. Trajectory Prediction Algorithm Based on Gaussian Mixture Model[J]. Journal of Software,2015,05:1048-1063.

[4] Dieng N A, Charbit M, Chaudet C, et al. Indoor Localization in Wireless Networks Based on a Two-Modes Gaussian Mixture Model[J]. 2013, 14(2382):1-5.

[5] Alfakih M, Keche M, and Benoudnine H. Gaussian mixture modeling for indoor positioning WIFI systems[C]. Control, Engineering \& Information Technology (CEIT), Tlemcen, 2015: 1-5.

[6] $\mathrm{Hu}$ Q. Methods to improve the applicability of WLAN-based indoor location estmation systems[J]. Journal of Huazhong University of Science \& Technology, 2013.

[7] Wang SW. Research on WLAN Indoor Location Method Based on Fingerprint [D]. Harbin Institute of Technology,2009.

[8] Pajovic M, Orlik P, et al. An Unsupervised Indoor Localization Method Based on Received Signal Strength (RSS) Measurements[C]. IEEE Global Communications Conference (GLOBECOM), San Diego, CA, 2015: 1-6.

[9] Xu Y B. WLAN Indoor Positioning Algorithm Based on KDDA and SVR[J]. Journal of Electronics \& Information Technology, 2011, 33(4):896-901.

[10] Zhang Y, Huang J, Xu K. Indoor positioning algorithm for WLAN based on principal component analysis and least square support vector regression[J]. Yi Qi Yi Biao Xue Bao/chinese Journal of Scientific Instrument, 2015, 36(2):408-414.

[11] Zhu Ji, et al. Multi-class adaboost. Statistics and its Interface, 2009, 2(3): 349-360.

[12] Sánchez-Rodríguez David, et al. A low complexity system based on multiple weighted decision trees for indoor localization. Sensors, 2015, 15(6): 14809-14829.

[13] FENG Yu, et al. Improved AdaBoost-based fingerprint algorithm for WiFi indoor localization. Information Technology and Artificial Intelligence Conference (ITAIC), 2014: 16-19.

[14] CANOVAS Oscar, et al. WiFiBoost: a terminal-based method for detection of indoor/outdoor places. In:Proceedings of the 11th International Conference on Mobile and Ubiquitous Systems: Computing, Networking and Services. ICST (Institute for Computer Sciences, Social-Informatics and Telecommunications Engineering), 2014. p. 352-353.

[15] Yang X, Zhuang M A, Yuan S. Multi-class Adaboost Algorithm Based on the Adjusted Weak Classifier[J]. Journal of Electronics \& Information Technology, 2016.

[16] Zhang BL. EM Algorithm and Simulation of Parameter Estimation in Finite Mixed Model [D]. Ningxia University,2015. 